\title{
Equivalence between the Existence of an Approximate Equilibrium in a Competitive Economy and Sperner's Lemma: A Constructive Analysis
}

\author{
Yasuhito Tanaka \\ Faculty of Economics, Doshisha University, Kamigyo-ku, Kyoto 602-8580, Japan \\ Correspondence should be addressed to Yasuhito Tanaka, yasuhito@mail.doshisha.ac.jp
}

Received 8 March 2011; Accepted 31 March 2011

Academic Editors: J. R. Fernandez and H.-T. Hu

Copyright (c) 2011 Yasuhito Tanaka. This is an open access article distributed under the Creative Commons Attribution License, which permits unrestricted use, distribution, and reproduction in any medium, provided the original work is properly cited.

\begin{abstract}
Brouwer's fixed point theorem cannot be constructively proved, so the existence of an equilibrium in a competitive economy also cannot be constructively proved. On the other hand, Sperner's lemma which is used to prove Brouwer's theorem is constructively proved. Some authors have presented a constructive (or an approximate) version of Brouwer's fixed point theorem using Sperner's lemma. In this paper, I prove the existence of an approximate equilibrium in a competitive economy directly by Sperner's lemma. Also I show that the existence of an approximate equilibrium leads to Sperner's lemma. I follow the Bishop style constructive mathematics according to Bishop and Bridges (1985), Bridges and Richman (1987), and Bridges and Vîţă (2006).
\end{abstract}

\section{Introduction}

It is often demonstrated that Brouwer's fixed point theorem cannot be constructively proved. Thus, the existence of an equilibrium in a competitive economy also cannot be constructively proved. On the other hand, however, Sperner's lemma which is used to prove Brouwer's theorem is constructively proved. Some authors have presented a constructive (or an approximate) version of Brouwer's fixed point theorem using Sperner's lemma. Thus, Brouwer's fixed point theorem can be constructively proved in its constructive version. See $[1,2]$. Using this theorem, we can prove the existence of an approximate equilibrium in a competitive economy. lemma?

Then, can we prove the existence of an approximate equilibrium directly by Sperner's 


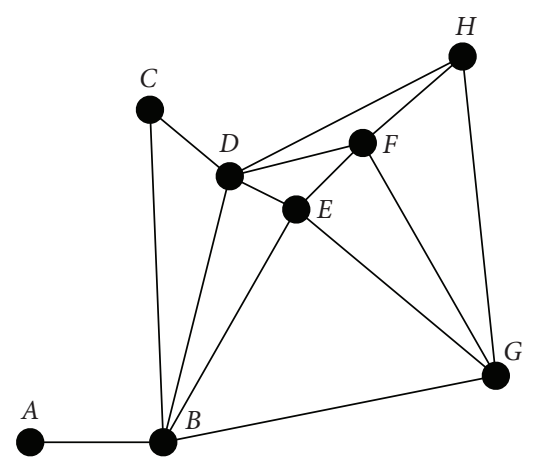

Figure 1: Example of graph.

This paper presents such a proof and also shows that the existence of an approximate equilibrium leads to Sperner's lemma. An approximate equilibrium in a competitive economy is a state such that excess demand for each good is smaller than $\varepsilon$ for each $\varepsilon>0$.

In the next section, the proof of Sperner's lemma will be presented. This proof is a standard constructive proof. In Section 3, the existence of an approximate equilibrium in a competitive exchange economy will be proved using Sperner's lemma. In Section 4 , it will be shown that the existence of an approximate equilibrium leads to Sperner's lemma. I follow the Bishop style constructive mathematics according to Bishop and Bridges [3], Bridges and Richman [4], and Bridges and Vîţă [5].

\section{Sperner's Lemma}

To prove Sperner's lemma, we use the following simple result of graph theory, the Handshaking lemma (For another constructive proof of Sperner's lemma, see [6].). A graph refers to a collection of vertices and a collection of edges that connect pairs of vertices. Each graph may be undirected or directed. Figure 1 is an example of an undirected graph. Degree of a vertex of a graph is defined to be the number of edges incident to the vertex, with loops counted twice. Each vertex has odd degree or even degree. Let $v$ denotes a vertex and $V$ denotes the set of all vertices.

Lemma 2.1 (the Handshaking lemma). Every undirected graph contains an even number of vertices of odd degree, that is, the number of vertices that have an odd number of incident edges must be even.

It is a simple lemma. But for completeness of arguments we provide a proof.

Proof. Prove this lemma by double counting. Let $d(v)$ be the degree of vertex $v$. The number of vertex-edge incidences in the graph may be counted in two different ways: by summing the degrees of the vertices or by counting two incidences for every edge. Therefore,

$$
\sum_{v \in V} d(v)=2 e
$$

where $e$ is the number of edges in the graph. The sum of the degrees of the vertices is therefore an even number. It could happen if and only if an even number of the vertices had odd degree. 


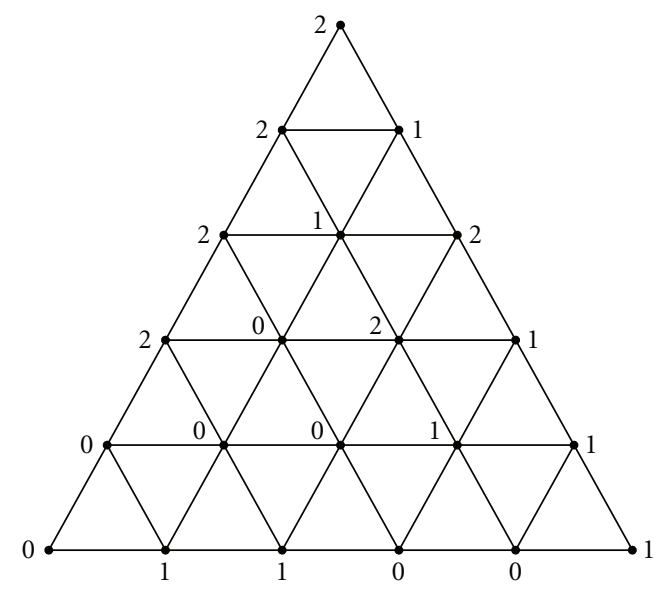

Figure 2: Partition and labeling of 2-dimensional simplex.

Let $\Delta$ denotes an $n$-dimensional simplex. $n$ is a finite natural number. For example, a 2-dimensional simplex is a triangle. Let us partition or triangulate the simplex. Figure 2 is an example of partition (triangulation) of a 2-dimensional simplex. In a 2-dimensional case, we divide each side of $\Delta$ in $m$ equal segments and draw the lines parallel to the sides of $\Delta . m$ is also a finite natural number. Then, the 2-dimensional simplex is partitioned into $m^{2}$ triangles. We consider partition of $\Delta$ inductively for cases of higher dimension. In a 3-dimensional case, each face of $\Delta$ is a 2-dimensional simplex, so it is partitioned into $m^{2}$ triangles in the way above mentioned, and draw the planes parallel to the faces of $\Delta$. Then, the 3-dimensional simplex is partitioned into $\mathrm{m}^{3}$ trigonal pyramids, and similarly for cases of higher dimension.

Let $K$ denotes the set of small $n$-dimensional simplices of $\Delta$ constructed by partition. Vertices of these small simplices of $K$ are labeled with the numbers $0,1,2, \ldots, n$ subject to the following rules:

(1) the vertices of $\Delta$ are respectively labeled 0 to $n$. We label a point $(1,0, \ldots, 0)$ with 0 , a point $(0,1,0, \ldots, 0)$ with 1 , a point $(0,0,1 \ldots, 0)$ with $2, \ldots$, a point $(0, \ldots, 0,1)$ with $n$, that is, a vertex whose $k$ th coordinate $(k=0,1, \ldots, n)$ is 1 and all other coordinates are 0 is labeled with $k$,

(2) if a vertex of $K$ is contained in an $n$-1-dimensional face of $\Delta$, then this vertex is labeled with some number which is the same as the number of a vertex of that face,

(3) if a vertex of $K$ is contained in an $n$-2-dimensional face of $\Delta$, then this vertex is labeled with some number which is the same as the number of a vertex of that face. And so on for cases of lower dimension,

(4) a vertex contained inside of $\Delta$ is labeled with an arbitrary number among $0,1, \ldots, n$.

A small simplex of $K$ which is labeled with the numbers $0,1, \ldots, n$ is called a fully labeled simplex. Now, let us prove Sperner's lemma.

Lemma 2.2 (Sperner's lemma). If one labels the vertices of $K$ following above rules (1) (4), then there are an odd number of fully labeled simplices. Thus, there exists at least one fully labeled simplex.

Proof. See the appendix. 


\section{Approximate Equilibrium in a Competitive Exchange Economy}

Consider a competitive exchange economy. There are $n+1$ goods $X_{0}, X_{1}, \ldots, X_{n} . n$ is a finite natural number. The prices of the goods are denoted by $p_{i}(\geq 0), i=0,1, \ldots, n$. Let $\bar{p}=p_{0}+$ $p_{1}+\cdots+p_{n}$, and define

$$
\bar{p}_{i}=\frac{p_{i}}{\bar{p}}, \quad i=0,1, \ldots, n
$$

Replace $\bar{p}_{0}, \bar{p}_{1}, \ldots, \bar{p}_{n}$, respectively, by $p_{0}, p_{1}, \ldots, p_{n}$. Then,

$$
p_{0}+p_{1}+\cdots+p_{n}=1
$$

Thus, $\mathbf{p}=\left(p_{0}, p_{1}, \ldots, p_{n}\right)$ represents a point on an $n$-dimensional simplex. Since consumers' demand (excess demand) for each good in a competitive exchange economy is determined by relative prices of the goods, such notation yields no loss of generality. We denote the vector of excess demands for the goods when the vector of prices is $\mathbf{p}$ by $\mathbf{f}(\mathbf{p})=\left(f_{1}, f_{2}, \ldots, f_{n}\right)$. Then, the following relation must hold:

$$
\mathbf{p} \mathbf{f}(\mathbf{p})=p_{0} f_{0}+p_{1} f_{1}+\cdots+p_{n} f_{n}=0(\text { Walras law })
$$

$f_{i}$ is equal to the sum of excess demands of all consumers for the good $X_{i}$. By the budget constraint for each consumer, in a competitive economy the sum of excess demands of all consumers for each good must be 0 . Adding the budget constraints for all consumers yields (3.3).

We assume that the excess demand function $\mathbf{f}(\mathbf{p})$ is uniformly continuous about the prices of the goods. Uniform continuity of $\mathbf{f}(\mathbf{p})$ means that for any $\mathbf{p}, \mathbf{p}^{\prime}$ and $\varepsilon>0$, there exists $\delta>0$ such that

$$
\text { If }\left|\mathbf{p}^{\prime}-\mathbf{p}\right|<\delta, \quad \text { then }\left|\mathbf{f}\left(\mathbf{p}^{\prime}\right)-\mathbf{f}(\mathbf{p})\right|<\varepsilon
$$

$\delta$ depends on neither $\mathbf{p}$ nor $\mathbf{p}^{\prime}$. Uniform continuity of demand functions implies that a slight price change yields only a slight demand change.

Consider the following function from the set of price vectors $\mathbf{p}=\left(p_{0}, p_{1}, \ldots, p_{n}\right)$ to the set of $n+1$ tuples of real numbers $\mathbf{v}=\left(v_{0}, v_{1}, \ldots, v_{n}\right)$ :

$$
v_{i}=p_{i}+\max \left(f_{i}, 0\right) \quad \forall i
$$


With this, we define a function from an $n$-dimensional simplex $\Delta$ to itself $\varphi(\mathbf{p})=\left(\varphi_{0}, \varphi_{1}, \ldots\right.$, $\left.\varphi_{n}\right)$ as follows:

$$
\varphi_{i}=\frac{1}{v_{0}+v_{1}+\cdots+v_{n}} v_{i}, \quad \forall i
$$

Since $\varphi_{i} \geq 0, i=0,1, \ldots, n$ and

$$
\varphi_{0}+\varphi_{1}+\cdots+\varphi_{n}=1
$$

$\left(\varphi_{0}, \varphi_{1}, \ldots, \varphi_{n}\right)$ represents a point on $\Delta$. By the uniform continuity of $\mathbf{f}, \varphi$ is also uniformly continuous.

Now, we show the following.

Theorem 3.1. In a competitive exchange economy, if the excess demand functions for the goods are uniformly continuous about their prices, then there exists an approximate equilibrium and one can constructively find the prices at the approximate equilibrium.

Proof. (1) First, we show that we can partition $\Delta$, which is the domain and range of $\varphi$, so that the conditions for Sperner's lemma are satisfied. We partition $\Delta$ according to the method in the proof of Sperner's lemma (see the appendix) and label the vertices of simplices constructed by partition of $\Delta$. It is important how to label the vertices contained in the faces of $\Delta$. Let $K$ be the set of small simplices constructed by partition of $\Delta, \mathbf{p}=\left(p_{0}, p_{1}, \ldots, p_{n}\right)$ a vertex of a simplex of $K$, and denote the $i$ th component of $\varphi(\mathbf{p})$ by $\varphi_{i}$. Then, we label a vertex $\mathrm{p}$ according to the following rule:

$$
\text { If } p_{k}>\varphi_{k} \quad \text { or } p_{k}+\tau>\varphi_{k} \text {, we label } \mathbf{p} \text { with } k \text {, }
$$

where $\tau$ is a positive number. If there are multiple $k^{\prime}$ s which satisfy this condition, we label $\mathbf{p}$ conveniently for the conditions of Sperner's lemma to be satisfied. We do not randomly label the vertices.

For example, let $\mathbf{p}$ be a point contained in an $n$-1-dimensional face of $\Delta$ such that $p_{i}=0$ for one $i$ among $0,1,2, \ldots, n$ (the $i$ th component of its coordinates is 0 ). With $\tau>0$, we have $\varphi_{i}>0$ or $\varphi_{i}<\tau$ (In constructive mathematics for any real number $x$, we cannot prove that $x \geq 0$ or $x<0$, nor that $x>0$ or $x=0$ or $x<0$. But for any distinct real numbers $x, y$ and $z$ such that $x>z$ we can prove that $x>y$ or $y>z$.). When $\varphi_{i}>0$, from $\sum_{j=0}^{n} p_{j}=1$, $\sum_{j=0}^{n} \varphi_{j}=1$ and $p_{i}=0$, we have

$$
\sum_{j=0, j \neq i}^{n} p_{j}>\sum_{j=0, j \neq i}^{n} \varphi_{j} .
$$

Then, for at least one $j$ (denote it by $k$ ), we have $p_{k}>\varphi_{k}$ and we label $\mathbf{p}$ with $k$, where $k$ is one of the numbers which satisfy $p_{k}>\varphi_{k}$. Since $\varphi_{i}>p_{i}, i$ does not satisfy this condition. When 
$\varphi_{i}<\tau, p_{i}=0$ implies $\sum_{j=0, j \neq i}^{n} p_{j}=1$. Since $\sum_{j=0, j \neq i}^{n} \varphi_{j} \leq 1$, we obtain

$$
\sum_{j=0, j \neq i}^{n} p_{j} \geq \sum_{j=0, j \neq i}^{n} \varphi_{j}
$$

Then, for a positive number $\tau$, we have

$$
\sum_{j=0, j \neq i}^{n}\left(p_{j}+\tau\right)>\sum_{j=0, j \neq i}^{n} \varphi_{j}
$$

Thus, there is at least one $j(\neq i)$ which satisfies $p_{j}+\tau>\varphi_{j}$. Denote it by $k$, and we label $\mathbf{p}$ with $k . k$ is one of the numbers other than $i$ such that $p_{k}+\tau>\varphi_{k}$ is satisfied. $i$ itself satisfies this condition $\left(p_{i}+\tau>\varphi_{i}\right)$. But, since there is a number other than $i$ which satisfies this condition, we can select a number other than $i$. We have proved that we can label the vertices contained in an $n$-1-dimensional face of $\Delta$ such that $p_{i}=0$ for one $i$ among $0,1,2, \ldots, n$ with the numbers other than $i$. By similar procedures, we can show that we can label the vertices contained in an $n$-2-dimensional face of $\Delta$ such that $p_{i}=0$ for two $i$ 's among $0,1,2, \ldots, n$ with the numbers other than those $i$ 's, and so on.

Consider the case where $p_{i}=p_{i+1}=0$. By similar procedures, we see that when $\varphi_{i}>0$ or $\varphi_{i+1}>0$,

$$
\sum_{j=0, j \neq i, i+1}^{n} p_{j}>\sum_{j=0, j \neq i, i+1}^{n} \varphi_{j} .
$$

Then, for at least one $j$ (denote it by $k$ ), we have $p_{k}>\varphi_{k}$ and we label $\mathbf{p}$ with $k$. On the other hand, when $\varphi_{i}<\tau$ and $\varphi_{i+1}<\tau$, we have

$$
\sum_{j=0, j \neq i, i+1}^{n} p_{j} \geq \sum_{j=0, j \neq i, i+1}^{n} \varphi_{j} .
$$

Then, for a positive number $\tau$, we have

$$
\sum_{j=0, j \neq i, i+1}^{n}\left(p_{j}+\tau\right)>\sum_{j=0, j \neq i, i+1}^{n} \varphi_{j} .
$$

There is at least one $j(\neq i, i+1)$ which satisfies $p_{j}+\tau>\varphi_{j}$. Denote it by $k$, and we label $\mathbf{p}$ with $k$.

Next, consider the case where $p_{i}=0$ for all $i$ other than $n$. If for some $i \varphi_{i}>0$, then we have $p_{n}>\varphi_{n}$, and label $\mathbf{p}$ with $n$. On the other hand, if $\varphi_{j}<\tau$ for all $j \neq n$, then we obtain $p_{n} \geq \varphi_{n}$. It implies $p_{n}+\tau>\varphi_{n}$. Thus, we can label $\mathbf{p}$ with $n$.

The conditions for Sperner's lemma are satisfied, and there exists an odd number of fully labeled simplices in $K$. 
(2) Suppose that we partition $\Delta$ sufficiently fine so that the distance between any pair of the vertices of small simplices is sufficiently small. Let $\mathbf{p}^{0}, \mathbf{p}^{1}, \ldots$, and $\mathbf{p}^{n}$ be the vertices of a fully labeled simplex. We name these vertices so that $\mathbf{p}^{0}, \mathbf{p}^{1}, \ldots, \mathbf{p}^{n}$ are labeled, respectively, with $0,1, \ldots, n$. The values of $\varphi$ at theses vertices are $\varphi\left(\mathbf{p}^{0}\right), \varphi\left(\mathbf{p}^{1}\right), \ldots$, and $\varphi\left(\mathbf{p}^{n}\right)$. The $i$ th components of $\mathbf{p}^{0}$ and $\varphi\left(\mathbf{p}^{0}\right)$ are denoted by $\mathbf{p}_{i}^{0}$ and $\varphi\left(\mathbf{p}^{0}\right)_{i}$, and so on.

The uniform continuity of $\varphi$ implies that we can select $\delta>0$ so that when the distance between $\mathbf{p}^{0}$ and $\mathbf{p}^{1}\left(\left|\mathbf{p}^{0}-\mathbf{p}^{1}\right|\right)$ is smaller than $\delta$, the distance between $\varphi\left(\mathbf{p}^{0}\right)$ and $\varphi\left(\mathbf{p}^{1}\right)\left(\mid \varphi\left(\mathbf{p}^{0}\right)-\right.$ $\left.\varphi\left(\mathbf{p}^{1}\right) \mid\right)$ is smaller than $\varepsilon$. We can make $\delta$ satisfying $\delta<\varepsilon$ (For example, for $\delta<1$ and $\varepsilon<1$, if when $\left|\mathbf{p}^{0}-\mathbf{p}^{1}\right|<\delta$ we have $\left|\varphi\left(\mathbf{p}^{0}\right)-\varphi\left(\mathbf{p}^{1}\right)\right|<\varepsilon$, then we have $\left|\varphi\left(\mathbf{p}^{0}\right)-\varphi\left(\mathbf{p}^{1}\right)\right|<\varepsilon$ also when $\left|\mathbf{p}^{0}-\mathbf{p}^{1}\right|<\delta \varepsilon<\varepsilon$.). Suppose $\tau>0$. About $\mathbf{p}^{0}$, from the labeling rules, we have $\mathbf{p}_{0}^{0}+\tau>\varphi\left(\mathbf{p}^{0}\right)_{0}$. About $\mathbf{p}^{1}$, also from the labeling rules we have $\mathbf{p}_{1}^{1}+\tau>\varphi\left(\mathbf{p}^{1}\right)_{1}$ which implies $\mathbf{p}_{1}^{1}>\varphi\left(\mathbf{p}^{1}\right)_{1}-\tau$. By the uniform continuity of $\varphi,\left|\mathbf{p}^{0}-\mathbf{p}^{1}\right|<\delta$ implies $\left|\varphi\left(\mathbf{p}^{0}\right)-\varphi\left(\mathbf{p}^{1}\right)\right|<\varepsilon$, which means $\varphi\left(\mathbf{p}^{1}\right)_{1}>$ $\varphi\left(\mathbf{p}^{0}\right)_{1}-\varepsilon$. On the other hand, $\left|\mathbf{p}^{0}-\mathbf{p}^{1}\right|<\delta$ means $\mathbf{p}_{1}^{0}>\mathbf{p}_{1}^{1}-\delta$. Thus, from

$$
\mathbf{p}_{1}^{0}>\mathbf{p}_{1}^{1}-\delta, \mathbf{p}_{1}^{1}>\varphi\left(\mathbf{p}^{1}\right)_{1}-\tau, \varphi\left(\mathbf{p}^{1}\right)_{1}>\varphi\left(\mathbf{p}^{0}\right)_{1}-\varepsilon,
$$

we obtain

$$
\mathbf{p}_{1}^{0}>\varphi\left(\mathbf{p}^{0}\right)_{1}-\delta-\varepsilon-\tau>\varphi\left(\mathbf{p}^{0}\right)_{1}-2 \varepsilon-\tau
$$

By similar arguments, for each $i$ other than 0 ,

$$
\mathbf{p}_{i}^{0}>\varphi\left(\mathbf{p}^{0}\right)_{i}-2 \varepsilon-\tau
$$

For $i=0$, we have $\mathbf{p}_{0}^{0}+\tau>\varphi\left(\mathbf{p}^{0}\right)_{0}$. Then,

$$
\mathbf{p}_{0}^{0}>\varphi\left(\mathbf{p}^{0}\right)_{0}-\tau
$$

Adding (3.17) and (3.18) side by side except for some $i$ (denote it by $k$ ) other than 0 yields

$$
\sum_{j=0, j \neq k}^{n} \mathbf{p}_{j}^{0}>\sum_{j=0, j \neq k}^{n} \varphi\left(\mathbf{p}^{0}\right)_{j}-2(n-1) \varepsilon-n \tau .
$$

From $\sum_{j=0}^{n} \mathbf{p}_{j}^{0}=1, \sum_{j=0}^{n} \varphi\left(\mathbf{p}^{0}\right)_{j}=1$, we have $1-\mathbf{p}_{k}^{0}>1-\varphi\left(\mathbf{p}^{0}\right)_{k}-2(n-1) \varepsilon-n \tau$, which is rewritten as

$$
\mathbf{p}_{k}^{0}<\varphi\left(\mathbf{p}^{0}\right)_{k}+2(n-1) \varepsilon+n \tau
$$

Since (3.17) implies $\mathbf{p}_{k}^{0}>\varphi\left(\mathbf{p}^{0}\right)_{k}-2 \varepsilon-\tau$, we have

$$
\varphi\left(\mathbf{p}^{0}\right)_{k}-2 \varepsilon-\tau<\mathbf{p}_{k}^{0}<\varphi\left(\mathbf{p}^{0}\right)_{k}+2(n-1) \varepsilon+n \tau .
$$


Thus,

$$
\left|\mathbf{p}_{k}^{0}-\varphi\left(\mathbf{p}^{0}\right)_{k}\right|<2(n-1) \varepsilon+n \tau
$$

is derived. On the other hand, adding (3.17) from 1 to $n$ yields

$$
\sum_{j=1}^{n} \mathbf{p}_{j}^{0}>\sum_{j=1}^{n} \varphi\left(\mathbf{p}^{0}\right)_{j}-2 n \varepsilon-n \tau
$$

From $\sum_{j=0}^{n} \mathbf{p}_{j}^{0}=1, \sum_{j=0}^{n} \varphi\left(\mathbf{p}^{0}\right)_{j}=1$, we have

$$
1-\mathbf{p}_{0}^{0}>1-\varphi\left(\mathbf{p}^{0}\right)_{0}-2 n \varepsilon-n \tau
$$

Then, from (3.18) and (3.24), we get

$$
\left|\mathbf{p}_{0}^{0}-\varphi\left(\mathbf{p}^{0}\right)_{0}\right|<2 n \varepsilon+n \tau
$$

Since $n$ is finite and $\varepsilon$ and $\tau$ are positive numbers which may be arbitrarily small, $2 n \varepsilon+n \tau$ and $2(n-1) \varepsilon+n \tau$ may also be arbitrarily small. Replacing $2 n \varepsilon+n \tau$ by $\varepsilon$, from (3.22) and (3.25) we obtain the following result:

$$
\left|\mathbf{p}_{i}^{0}-\varphi\left(\mathbf{p}^{0}\right)_{i}\right|<\varepsilon \quad \forall i
$$

All points contained in the fully labeled simplex of $K$ satisfy this relation. have

(3) Denote a point which satisfies (3.26) by $\widetilde{\mathbf{p}}$, and denote its $i$ th component by $\tilde{p}_{i}$. We

$$
\left|\tilde{p}_{i}-\varphi_{i}\right|<\varepsilon \quad \forall i
$$

Let us consider the relationship between the price and demand for each good in that case. From the definitions of $\varphi$ and $\mathbf{v}$, and $\sum_{j=0}^{n} \boldsymbol{v}_{j}=1+\sum_{j=0}^{n} \max \left(f_{j}, 0\right)$ (because $\sum_{j=0}^{n} \tilde{p}_{j}=1$ ), (3.27) means

$$
\left|\frac{\tilde{p}_{i}+\max \left(f_{i}, 0\right)}{1+\sum_{j=0}^{n} \max \left(f_{j}, 0\right)}-\tilde{p}_{i}\right|=\left|\frac{\max \left(f_{i}, 0\right)-\tilde{p}_{i} \sum_{j=0}^{n} \max \left(f_{j}, 0\right)}{1+\sum_{j=0}^{n} \max \left(f_{j}, 0\right)}\right|<\varepsilon
$$

Let $\lambda=\sum_{j=0}^{n} \max \left(f_{\mathrm{j}}, 0\right)$. Then, we have $\left|\left(\max \left(f_{i}, 0\right)-\lambda \tilde{p}_{i}\right) /(1+\lambda)\right|<\varepsilon$. It is rewritten as

$$
\left|\max \left(f_{i}, 0\right)-\lambda \tilde{p}_{i}\right|<(1+\lambda) \varepsilon
$$


This means

$$
-(1+\lambda) \varepsilon+\lambda \tilde{p}_{i}<\max \left(f_{i}, 0\right)<(1+\lambda) \varepsilon+\lambda \tilde{p}_{i}
$$

From $\sum_{j=0}^{n} \tilde{p}_{j}=1$, there is a $k$ which satisfies $\tilde{p}_{k}>0$. If for all such $k \max \left(f_{k}, 0\right)=f_{k}>0$ holds, that is, excess demands for all goods with positive prices are positive, we cannot cancel out $\tilde{p}_{k} f_{k}>0$ because the price of any good cannot be negative and the Walras law (3.3) is violated (Even if we relax the Walras law to the following approximate Walras law, we can show essentially the same result: $|\mathbf{p f}(\mathbf{p})|=\left|p_{0} f_{0}+p_{1} f_{1}+\cdots+p_{n} f_{n}\right|<\varepsilon$, where $\varepsilon$ is a positive number. If we have $\max \left(f_{k}, 0\right)=f_{k}>0$ for all $k$ which satisfy $p_{k}>0$, this inequality cannot be satisfied because the price of any good cannot be negative.). Therefore, $\lambda$ as well as $\varepsilon$ must be a positive number which may be arbitrarily small, and since $\tilde{p}_{i}$ is finite, $(1+\lambda) \varepsilon+\lambda \tilde{p}_{i}$ is a real number which may also be arbitrarily small. There exists a number which is only slightly larger than $(1+\lambda) \varepsilon+\lambda \tilde{p}_{i}$. Replace $(1+\lambda) \varepsilon+\lambda \tilde{p}_{i}$ by such a number, and denote it by $\varepsilon$. Then, for all $i$ we have

$$
\max \left(f_{i}, 0\right)<\varepsilon
$$

This means that excess demand for each good is smaller than $\varepsilon$. Such a state is an approximate equilibrium. In the approximate equilibrium when $p_{i}>0$, we must have $f_{i}>-\varepsilon$ for any $\varepsilon>0$ because if $f_{i}<0$ we have $p_{i} f_{i}<0$, and then the Walras law is violated. On the other hand, there may be excess supply (negative excess demand) for a good whose price is zero.

\section{From the Existence of an Approximate Equilibrium to Sperner's Lemma}

In this section, we will derive Sperner's lemma from the existence of an approximate equilibrium in a competitive economy. Let us partition an $n$-dimensional simplex $\Delta$. Denote the set of small $n$-dimensional simplices of $\Delta$ constructed by partition by $K$. Vertices of these small simplices of $K$ are labeled with the numbers $0,1,2, \ldots, n$ similarly to the proof of Sperner's lemma. Denote vertices of an $n$-dimensional simplex of $K$ by $x^{0}, x^{1}, \ldots, x^{n}$, the $j$ th component of $x^{i}$ by $x_{j}^{i}$, and the label of $x^{i}$ by $l\left(x^{i}\right)$. Let $\tau$ be a positive number which is smaller than $x_{l\left(x^{i}\right)}^{i}$ for all $x^{i}$, and define a function $f\left(x^{i}\right)$ as follows:

$$
\begin{gathered}
f\left(x^{i}\right)=\left(f_{0}\left(x^{i}\right), f_{1}\left(x^{i}\right), \ldots, f_{n}\left(x^{i}\right)\right), \\
f_{j}\left(x^{i}\right)= \begin{cases}x_{j}^{i}-\tau & \text { for } j=l\left(x^{i}\right), \\
x_{j}^{i}+\frac{\tau}{n} & \text { for } j \neq l\left(x^{i}\right) .\end{cases}
\end{gathered}
$$

$f_{j}$ denotes the $j$ th component of $f$. From the labeling rules $x_{l\left(x^{i}\right)}^{i}>0$ for all $x^{i}$, and so $\tau>0$ is well defined. Since $\sum_{j=0}^{n} f_{j}\left(x^{i}\right)=\sum_{j=0}^{n} x_{j}^{i}=1$, we have

$$
f\left(x^{i}\right) \in \Delta
$$


We extend $f$ to all points in the simplex by convex combinations of its values on the vertices of the simplex. Let $y$ be a point in the $n$-dimensional simplex of $K$ whose vertices are $x^{0}, x^{1}, \ldots, x^{n}$. Then, $y$ and $f(y)$ are represented as follows:

$$
y=\sum_{i=0}^{n} \lambda_{i} x^{i}, \quad f(y)=\sum_{i=0}^{n} \lambda_{i} f\left(x^{i}\right), \quad \lambda_{i} \geq 0, \sum_{i=0}^{n} \lambda_{i}=1
$$

Let us show that $f$ is uniformly continuous. Let $y$ and $y^{\prime}$ be distinct points in the same small $n$-dimensional simplex of $K$. They are represented as

$$
y=\sum_{i=0}^{n} \lambda_{i} x^{i}, \quad y^{\prime}=\sum_{i=0}^{n} \lambda_{i}^{\prime} x^{i}
$$

and so

$$
y-y^{\prime}=\sum_{i=0}^{n}\left(\lambda_{i}-\lambda_{i}^{\prime}\right) x^{i}, \quad y_{j}-y_{j}^{\prime}=\sum_{i=0}^{n}\left(\lambda_{i}-\lambda_{i}^{\prime}\right) x_{j}^{i} \quad \text { for each } j .
$$

Then, we have

$$
f(y)-f\left(y^{\prime}\right)=\sum_{i=0}^{n}\left(\lambda_{i}-\lambda_{i}^{\prime}\right) f\left(x^{i}\right)
$$

and for each $j$,

$$
\begin{aligned}
f_{j}(y)-f_{j}\left(y^{\prime}\right) & =\sum_{i=0}^{n}\left(\lambda_{i}-\lambda_{i}^{\prime}\right) x_{j}^{i}+\sum_{i: j \neq l(i)}\left(\lambda_{i}-\lambda_{i}^{\prime}\right) \frac{\tau}{n}-\sum_{i: j=l(i)}\left(\lambda_{i}-\lambda_{i}^{\prime}\right) \tau \\
& =y_{j}-y_{j}^{\prime}+\sum_{i: j \neq l(i)}\left(\lambda_{i}-\lambda_{i}^{\prime}\right) \frac{\tau}{n}-\sum_{i: j=l(i)}\left(\lambda_{i}-\lambda_{i}^{\prime}\right) \tau .
\end{aligned}
$$

Since $\tau$ is finite, appropriately selecting $\lambda_{i}^{\prime}$ given $\lambda_{i}$ for each $i$, we can make $\left|f_{j}(y)-f_{j}\left(y^{\prime}\right)\right|$ sufficiently small corresponding to the value of $\left|y_{j}-y_{j}^{\prime}\right|$ for each $j$, and so make $\left|f(y)-f\left(y^{\prime}\right)\right|$ sufficiently small corresponding to the value of $\left|y-y^{\prime}\right|$. Thus, $f$ is uniformly continuous.

Now, using $f$, we construct an excess demand function as follows:

$$
z_{i}(\mathbf{p})=f_{i}(\mathbf{p})-p_{i} \mu(\mathbf{p}), \quad i=0,1, \ldots, n
$$

$\mathbf{p} \in \Delta$ and $\mu(\mathbf{p})$ is defined by

$$
\mu(\mathbf{p})=\frac{\sum_{i=0}^{n} p_{i} f_{i}(\mathbf{p})}{\sum_{i=0}^{n} p_{i}^{2}} .
$$


Each $z_{i}(\mathbf{p})$ is uniformly continuous, and satisfies the Walras law as shown below. Multiplying $p_{i}$ (the $i$ th component of $\mathbf{p}$ ) to (4.9) for each $i$ and adding them from 0 to $n$ yields

$$
\begin{aligned}
\sum_{i=0}^{n} p_{i} z_{i} & =\sum_{i=0}^{n} p_{i} f_{i}(\mathbf{p})-\mu(\mathbf{p}) \sum_{i=0}^{n} p_{i}^{2}=\sum_{i=0}^{n} p_{i} f_{i}(\mathbf{p})-\frac{\sum_{i=0}^{n} p_{i} f_{i}(\mathbf{p})}{\sum_{i=0}^{n} p_{i}^{2}} \sum_{i=0}^{n} p_{i}^{2} \\
& =\sum_{i=0}^{n} p_{i} f_{i}(\mathbf{p})-\sum_{i=0}^{n} p_{i} f_{i}(\mathbf{p})=0 .
\end{aligned}
$$

Therefore, $z_{i}(\mathbf{p})$ 's satisfy the conditions for excess demand functions and there exists an approximate equilibrium. Let $\mathbf{p}^{*}=\left\{p_{0}^{*}, p_{1}^{*}, \ldots, p_{n}^{*}\right\}$ be the price vector at the approximate equilibrium. Then, from $\max \left(z_{i}, 0\right)<\varepsilon$ (see (3.31)), we have $f_{i}\left(\mathbf{p}^{*}\right)-p_{i}^{*} \mu\left(\mathbf{p}^{*}\right)<\varepsilon$ for all $i$, where $\varepsilon$ is an positive number. Since it is impossible that at the approximate equilibrium $z_{i}<0$ for $i$ satisfying $p_{i}^{*}>0$ because of (4.11), we have $z_{i}=f_{i}\left(\mathbf{p}^{*}\right)-p_{i}^{*} \mu\left(\mathbf{p}^{*}\right)>-\varepsilon$ for such $i$. On the other hand, for $i$ such that $p_{i}^{*}=0$, we have $z_{i}=f_{i}\left(\mathbf{p}^{*}\right) \geq 0$. Therefore,

$$
-\varepsilon<f_{i}\left(\mathbf{p}^{*}\right)-p_{i}^{*} \mu\left(\mathbf{p}^{*}\right)<\varepsilon
$$

is obtained. Adding this inequality side by side from 0 to $n$ yields

$$
-(n+1) \varepsilon<\sum_{i=0}^{n} f_{i}\left(\mathbf{p}^{*}\right)-\mu\left(\mathbf{p}^{*}\right) \sum_{i=0}^{n} p_{i}^{*}<(n+1) \varepsilon
$$

From $\sum_{i=0}^{n} f_{i}\left(\mathbf{p}^{*}\right)=\sum_{i=0}^{n} p_{i}^{*}=1$, we obtain

$$
1-(n+1) \varepsilon<\mu\left(\mathbf{p}^{*}\right)<1+(n+1) \varepsilon .
$$

Further, from (4.12) and (4.14), we get

$$
p_{i}^{*}-(n+1) \varepsilon p_{i}^{*}-\varepsilon<f_{i}\left(\mathbf{p}^{*}\right)<p_{i}^{*}+(n+1) \varepsilon p_{i}^{*}+\varepsilon .
$$

Since $n$ and $p_{i}^{*}$ are finite, $(n+1) \varepsilon p_{i}^{*}+\varepsilon$ is a positive real number which may be arbitrarily small. There exists a number which is only slightly larger than $(n+1) \varepsilon p_{i}^{*}+\varepsilon$. Replace $(n+1) \varepsilon p_{i}^{*}+\varepsilon$ by such a number, and denote it by $\varepsilon$. Then, $-\varepsilon<f_{i}\left(\mathbf{p}^{*}\right)-p_{i}^{*}<\varepsilon$, that is,

$$
\left|f_{i}\left(\mathbf{p}^{*}\right)-p_{i}^{*}\right|<\varepsilon
$$

is derived. This relation holds for all $i$. 
Let $\gamma>0$ and $\widetilde{\mathbf{p}}$ be a point in $V\left(\mathbf{p}^{*}, \gamma\right)$, where $V\left(\mathbf{p}^{*}, \gamma\right)$ is a $\gamma$-neighborhood of $\mathbf{p}^{*}$. If $\gamma$ is sufficiently small, uniform continuity of $f$ means

$$
\left|f_{i}(\tilde{\mathbf{p}})-\widetilde{\mathbf{p}}_{i}\right|<\varepsilon,
$$

for any $\varepsilon>0$ and for all $i$. $\tilde{\mathbf{p}}_{i}$ is the $i$ th component of $\tilde{\mathbf{p}}$. Let $\Delta^{*}$ be a simplex of $K$ which contains $\tilde{\mathbf{p}}$, and $\mathbf{p}^{0}, \mathbf{p}^{1}, \ldots, \mathbf{p}^{n}$ be the vertices of $\Delta^{*}$. Then, $\tilde{\mathbf{p}}$ and $f(\tilde{\mathbf{p}})$ are represented as

$$
\widetilde{\mathbf{p}}=\sum_{i=0}^{n} \lambda_{i} \mathbf{p}^{i}, \quad f(\widetilde{\mathbf{p}})=\sum_{i=0}^{n} \lambda_{i} f\left(\mathbf{p}^{i}\right), \quad \lambda_{i} \geq 0, \sum_{i=0}^{n} \lambda_{i}=1
$$

Equation (4.2) implies that if only one $\mathbf{p}^{k}$ among $\mathbf{p}^{0}, \mathbf{p}^{1}, \ldots, \mathbf{p}^{n}$ is labeled with $i$, we have

$$
\begin{aligned}
\left|f_{i}(\tilde{\mathbf{p}})-\tilde{\mathbf{p}}_{i}\right| & =\left|\sum_{j=0}^{n} \lambda_{j} \mathbf{p}_{i}^{j}+\sum_{j=0, j \neq k} \lambda_{j} \frac{\tau}{n}-\lambda_{k} \tau-\tilde{\mathbf{p}}_{i}\right| \\
& =\left|\left(\frac{1}{n} \sum_{j=0, j \neq k} \lambda_{j}-\lambda_{k}\right) \tau\right|<\varepsilon .
\end{aligned}
$$

$\mathbf{p}_{i}^{j}$ is the $i$ th component of $\mathbf{p}^{j}$.

Since $\varepsilon$ may be arbitrarily small and $\tau>0$, this means

$$
\frac{1}{n} \sum_{j=0, j \neq k} \lambda_{j}-\lambda_{k} \approx 0
$$

Equation (4.17) is satisfied with $\lambda_{k} \approx 1 /(n+1)$ for all $k$. On the other hand if no $\mathbf{p}^{j}$ is labeled with $i$, we have

$$
f_{i}(\tilde{\mathbf{p}})=\sum_{j=0}^{n} \lambda_{j} \mathbf{p}_{i}^{j}=\mathbf{p}_{i}^{*}+\left(1+\frac{1}{n}\right) \tau,
$$

and then (4.17) cannot be satisfied. Thus, for each $i$ one and only one $\mathbf{p}^{j}$ must be labeled with $i$. Therefore, $\Delta^{*}$ must be a fully labeled simplex. We have completed the proof of Sperner's lemma.

\section{Concluding Remark}

In this paper, I have presented a proof of the existence of an approximate equilibrium in a competitive economy directly by Sperner's lemma from a viewpoint of constructive mathematics. In another paper [7], I apply this method to prove the existence of an approximate Nash equilibrium in a finite strategic game (a strategic game with a finite number of players and a finite number of pure strategies). 


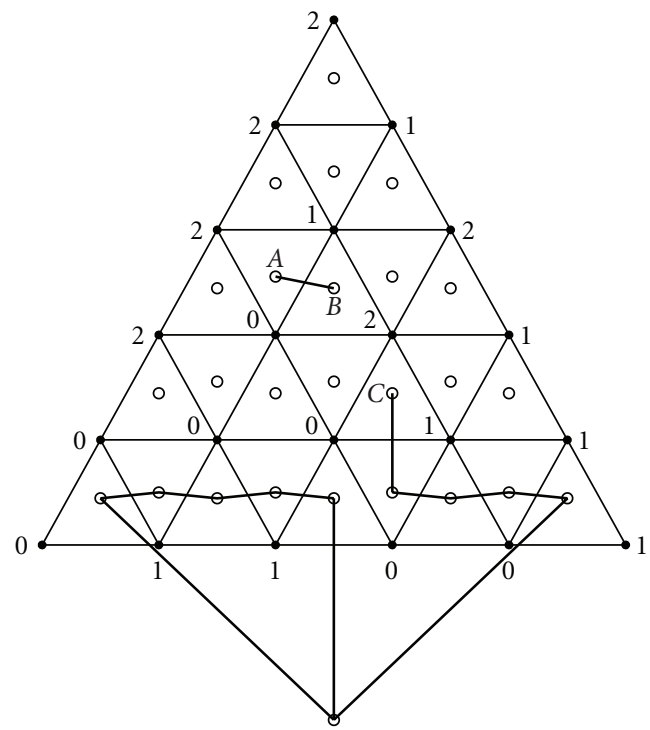

Figure 3: Sperner's lemma.

\section{Appendix}

\section{Proof of Sperner's Lemma}

We prove this lemma by induction about the dimension of $\Delta$. When $n=0$, we have only one point with the number 0 . It is the unique 0 -dimensional simplex. Therefore, the lemma is trivial. When $n=1$, a partitioned 1-dimensional simplex is a segmented line. The endpoints of the line are labeled distinctly, by 0 and 1 . Hence, in moving from endpoint 0 to endpoint 1 , the labeling must switch an odd number of times, that is, an odd number of edges labeled with 0 and 1 may be located in this way.

Next, consider the case of 2 dimension. Assume that we have partitioned a 2dimensional simplex (triangle) $\Delta$ as explained above. Consider the face of $\Delta$ labeled with 0 and 1 (We call edges of triangle $\Delta$ faces to distinguish between them and edges of a dual graph which we will consider later.). It is the base of the triangle in Figure 3. Now, we introduce a dual graph that has its nodes in each small triangle of $K$ plus one extra node outside the face of $\Delta$ labeled with 0 and 1 (putting a dot in each small triangle and one dot outside $\Delta$ ). We define edges of the graph that connect two nodes if they share a side labeled with 0 and 1 . See Figure 3. White circles are nodes of the graph, and thick lines are its edges. Since from the result of 1-dimensional case there are an odd number of faces of $K$ labeled with 0 and 1 contained in the face of $\Delta$ labeled with 0 and 1 , there are an odd number of edges which connect the outside node and inside nodes. Thus, the outside node has odd degree. Since by the Handshaking lemma there are an even number of nodes which have odd degree, we have at least one node inside the triangle which has odd degree. Each node of our graph except for the outside node is contained in one of small triangles of $K$. Therefore, if a small triangle of $K$ has one face labeled with 0 and 1 , the degree of the node in that triangle is 1 ; if a small triangle of $K$ has two such faces, the degree of the node in that triangle is 2 , and if a small triangle of $K$ has no such face, the degree of the node in that triangle is 0 . Thus, if the degree 
of a node is odd, it must be 1 and then the small triangle which contains this node is labeled with 0,1 and 2 (fully labeled). In Figure 3, triangles which contain one of the nodes $A, B, C$ are fully labeled triangles.

Now, assume that the theorem holds for dimensions up to $n-1$. Assume that we have partitioned an $n$-dimensional simplex $\Delta$. Consider the fully labeled face of $\Delta$ which is a fully labeled $n$-1-dimensional simplex. Again, we introduce a dual graph that has its nodes in small $n$-dimensional simplices of $K$ plus one extra node outside the fully labeled face of $\Delta$ (putting a dot in each small $n$-dimensional simplex and one dot outside $\Delta$ ). We define the edges of the graph that connect two nodes if they share a face labeled with $0,1, \ldots, n-1$. Since from the result of $n$-1-dimensional case there are an odd number of fully labeled faces of small simplices of $K$ contained in the $n$-1-dimensional fully labeled face of $\Delta$, there are an odd number of edges which connect the outside node and inside nodes. Thus, the outside node has odd degree. Since, by the Handshaking lemma there are an even number of nodes which have odd degree, we have at least one node inside the simplex which has odd degree. Each node of our graph except for the outside node is contained in one of small $n$-dimensional simplices of $K$. Therefore, if a small simplex of $K$ has one fully labeled face, the degree of the node in that simplex is 1 ; if a small simplex of $K$ has two such faces, the degree of the node in that simplex is 2, and if a small simplex of $K$ has no such face, the degree of the node in that simplex is 0 . Thus, if the degree of a node is odd, it must be 1 and then the small simplex which contains this node is fully labeled.

If the number (label) of a vertex other than vertices labeled with $0,1, \ldots, n-1$ of an $n$-dimensional simplex which contains a fully labeled $n$-1-dimensional face is $n$, then this $n$ dimensional simplex has one such face, and this simplex is a fully labeled $n$-dimensional simplex. On the other hand, if the number of that vertex is other than $n$, then the $n$ dimensional simplex has two such faces.

We have completed the proof of Sperner's lemma.

Since $n$ and partition of $\Delta$ are finite, the number of small simplices constructed by partition is also finite. Thus, we can constructively find a fully labeled $n$-dimensional simplex of $K$ through finite steps.

\section{Acknowledgments}

The author thanks the anonymous referees for their very useful comments. This research was partially supported by the Ministry of Education, Science, Sports and Culture of Japan, Grantin-Aid for Scientific Research (C), no. 20530165, and the Special Costs for Graduate Schools of the Special Expenses for Hitech Promotion by the Ministry of Education, Science, Sports and Culture of Japan in 2010.

\section{References}

[1] D. van Dalen, "Brouwer's $\varepsilon$-fixed point from Sperner's lemma," Logic Group Preprint Series, vol. 275, pp. 1-20, 2009.

[2] W. Veldman, "Brouwer's approximate fixed-point theorem is equivalent to Brouwer's fan theorem," in Logicism, intuitionism, and formalism, S. Lindström, E. Palmgren, K. Segerberg, and S. Hansen, Eds., vol. 341, pp. 277-299, Springer, Dordrecht, The Netherlands, 2009.

[3] E. Bishop and D. Bridges, Constructive Analysis, vol. 279, Springer, Berlin, Germany, 1985.

[4] D. Bridges and F. Richman, Varieties of Constructive Mathematics, vol. 97 of London Mathematical Society Lecture Note Series, Cambridge University Press, Cambridge, UK, 1987. 
[5] D. S. Bridges and L. S. Viţ̧̆ă, Techniques of Constructive Analysis, Springer, New York, NY, USA, 2006.

[6] F. E. Su, "Rental harmony: Sperner's lemma in fair division," The American Mathematical Monthly, vol. 106, no. 10, pp. 930-942, 1999.

[7] Y. Tanaka, "A proof of the existence of an approximate Nash equilibrium in a finite strategic game directly by Sperner's lemma: a constructive analysis," Mimeograph. In press.

[8] J. Nash, “Non-cooperative games," Annals of Mathematics, vol. 54, pp. 286-295, 1951. 


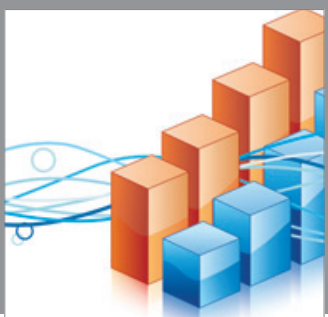

Advances in

Operations Research

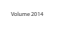

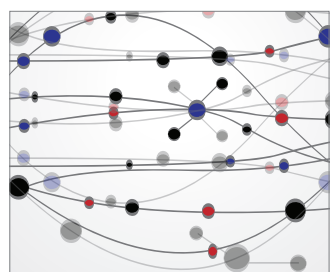

\section{The Scientific} World Journal
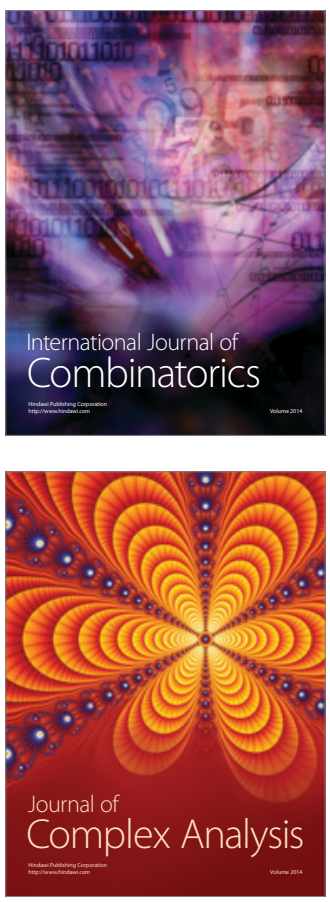

International Journal of

Mathematics and

Mathematical

Sciences
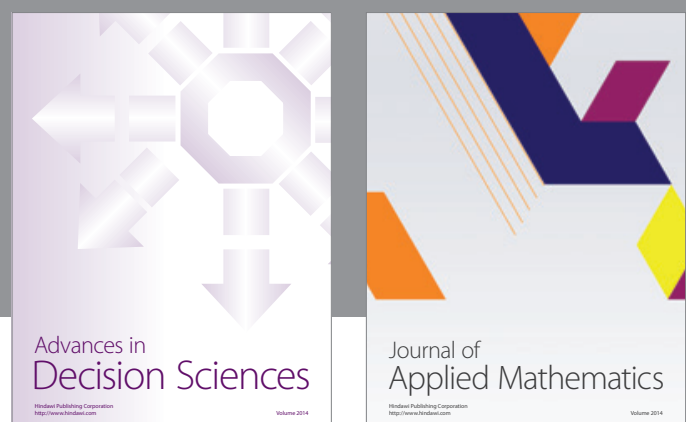

Journal of

Applied Mathematics
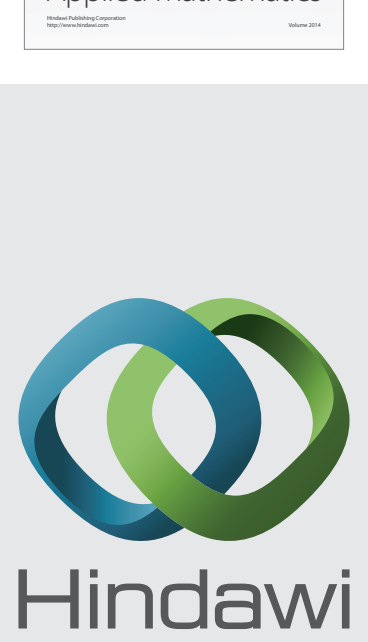

Submit your manuscripts at http://www.hindawi.com
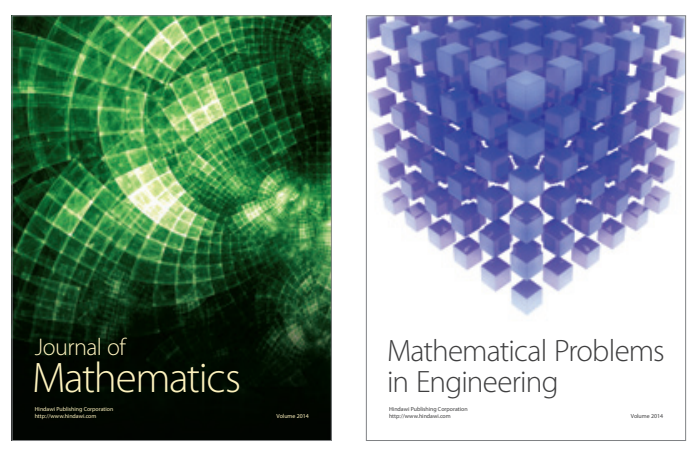

Mathematical Problems in Engineering
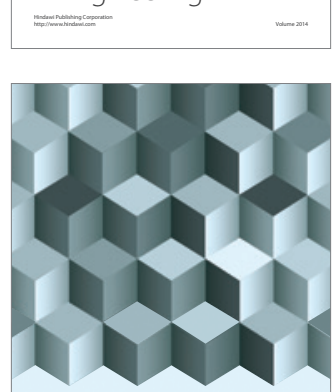

Journal of

Function Spaces
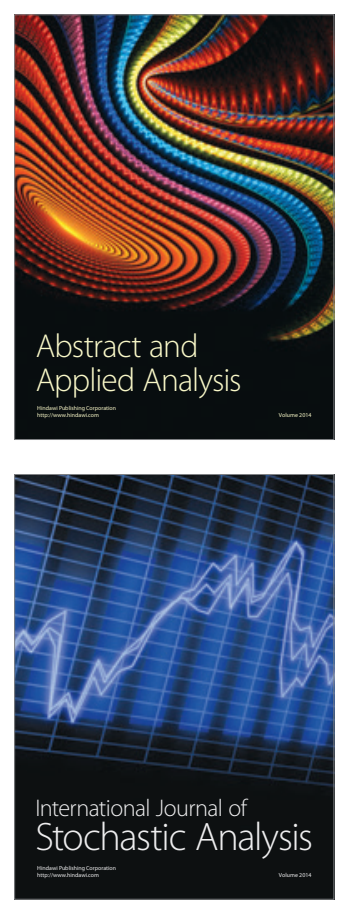

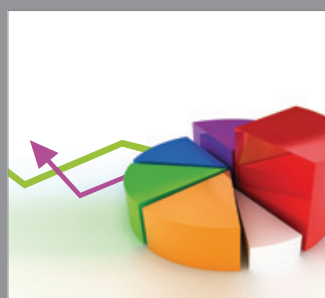

ournal of

Probability and Statistics

Promensencen
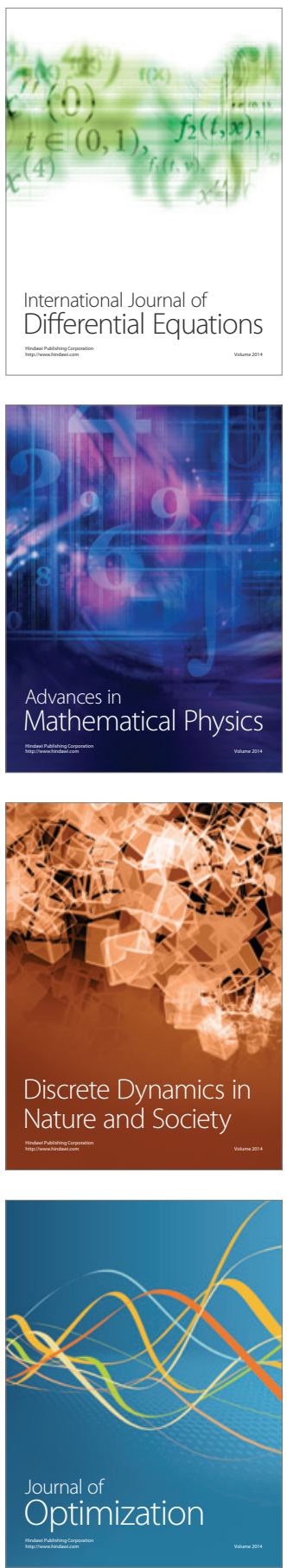